\title{
Diagnostic accuracy of MRI to evaluate tumour response and residual tumour size after neoadjuvant chemotherapy in breast cancer patients
}

\author{
Alberto Bouzón'1, Benigno Acea1 ${ }^{1}$, Rafaela Soler², Ángela Iglesias², Paz Santiago³, \\ Joaquín Mosquera ${ }^{2}$, Lourdes Calvo ${ }^{4}$, Teresa Seoane-Pillado ${ }^{5}$, Alejandra García ${ }^{1}$ \\ ${ }^{1}$ Department of Surgery; Breast Unit. Complexo Hospitalario Universitario de A Coruña Sergas, Spain \\ ${ }^{2}$ Department of Radiology, Breast Unit. Complexo Hospitalario Universitario de A Coruña Sergas, Spain \\ ${ }^{3}$ Department of Anatomic Pathology, Breast Unit. Complexo Hospitalario Universitario de A Coruña Sergas, Spain \\ ${ }^{4}$ Department of Clinical Oncology, Breast Unit. Complexo Hospitalario Universitario de A Coruña Sergas, Spain \\ ${ }^{5}$ Clinical Epidemiology and Biostatistics Unit, Breast Unit. Complexo Hospitalario Universitario de A Coruña Sergas, Spain
}

Radiol Oncol 2016; 50(1): 73-79.

Received 30 September 2015

Accepted 30 December 2015

Correspondence to: Alberto Bouzón, M.D., Department of Surgery, Breast Unit, Hospital Abente y Lago, Rd: Gral Sir John Moore, ${ }^{\circ} 1$, A Coruña, Spain. Phone: +34 690103810; Email: dr.alberto@aecirujanos.es

Disclosure: No potential conflicts of interest were disclosed.

Background. The aim, of the study was to estimate the accuracy of magnetic resonance imaging (MRI) in assessing residual disease in breast cancer patients receiving neoadjuvant chemotherapy (NAC) and to identify the clinicopathological factors that affect the diagnostic accuracy of breast MRI to determine residual tumour size following NAC.

Patients and methods. 91 breast cancer patients undergoing NAC (92 breast lesions) were included in the study. Breast MRI was performed at baseline and after completion of NAC. Treatment response was evaluated by MRI and histopathological examination to investigate the ability of MRI to predict tumour response. Residual tumour size was measured on post-treatment MRI and compared with pathology in 89 lesions. Clinicopathological factors were analyzed to compare MRI-pathologic size differences.

Results. The overall sensitivity, specificity, positive predictive value, negative predictive value, and accuracy for diagnosing invasive residual disease by using MRI were $75.00 \%, 78.57 \%, 88.89 \%, 57.89 \%$, and $76.09 \%$ respectively. The Pearson's correlation coefficient ( $r$ ) between tumour sizes determined by MRI and pathology was $r=0.648(p<0.001)$. The size discrepancy was significantly lower in cancers with initial MRI size $\leq 5 \mathrm{~cm}(p=0.050)$, in cancers with high tumour grade $(p<0.001)$, and in patients with hormonal receptor-negative cancer $(p=0.033)$.

Conclusions. MRI is an accurate tool for evaluating tumour response after NAC. The accuracy of MRI in estimating residual tumour size varies with the baseline MRI tumour size, the tumour grade and the hormonal receptor status.

Key words: breast cancer; MRI; residual tumour; neoadjuvant chemotherapy

\section{Introduction}

Neoadjuvant chemotherapy (NAC) has been used in the management of large operable breast tumours with the purpose of modifying the surgical planning and increase the rate of breast conserva- tive surgery (BCS) ${ }^{1-6}$ Currently, NAC has been extended to selected patients with early-stage disease to improve the cosmetic outcome of BCS, especially in women with small breast size. ${ }^{7-9}$ NAC has proved to be equivalent to postoperative chemotherapy in terms of disease-free and overall surviv- 
al. ${ }^{10-12}$ However, in the neoadjuvant setting, there is evidence that patients who achieve a pathologic complete response (pCR) in the breast after NAC have a better prognosis than patients with a partial response or non-responders.6,11,13-15 Based on the different behaviour of each tumour subtype, a molecular classification system identifies subgroups of breast invasive carcinoma patients who are most likely to achieve a pCR. ${ }^{16}$

An accurate imaging assessment of tumour response to NAC may facilitate the surgical planning. Magnetic resonance imaging (MRI) is more accurate and sensitive than conventional methods in assessing residual tumour extent after NAC. ${ }^{17-23}$ In addition; there has been a positive correlation between MRI-determined and pathologic residual tumour size. ${ }^{24-29}$ However, it is necessary to know the factors affecting the diagnostic accuracy of MRI in breast cancer treated with NAC.

The aims of the present study are to evaluate the diagnostic accuracy of MRI to detect residual disease and to predict the tumour extent in patients with breast cancer receiving NAC, and to identify the factors that influence the accuracy of MRI in predicting residual tumour size.

\section{Patients and methods}

\section{Patients and tumour molecular characteristics}

A total of 91 patients with invasive breast cancer (92 carcinomas) were included in this institutional retrospective study. All patients were diagnosed by core needle biopsy between October 2006 and June 2013. All patients were treated with NAC followed by surgical treatment and underwent MRI before and after NAC for monitoring tumour response to treatment. Considering the Helsinki Declaration principles, the Institutional Research Ethics Committee approved this study (No. 2015/059).

According to the results of the initial diagnostic biopsy, tumours were classified into 5 molecular subtypes based on immunohistochemical characteristics of breast cancer ${ }^{30}$ (hormone receptor status, human epidermal growth factor receptor 2 (HER2) status and level of expression of ki-67). Estrogen receptor (ER) and progesterone receptor (PR) were considered positive if $\geq 10 \%$ of tumour cell nuclei stained positive. Hormone receptors (HR) were considered positive when the ER, PR, or both were positive. HER2 tumours scoring 3+ (intense homogeneous membranous staining in $\geq 10 \%$ of tumour cells) were considered positive. In case of $2+$ scores (moderate complete membranous staining in $\geq 10 \%$ of tumour cells), the technique of fluorescent in situ hybridization was used to determine HER2 gene amplification. Samples with scores 0 to $1+$ were considered negative. The cut-off of ki-67 expression level was set at $14 \%$, to determine whether the cell proliferation index was high $(>14 \%)$ or low $(\leq 14 \%)$. The five categories of molecular subtypes were: $l u$ minal A-like subtype (HR positive, HER2 negative, ki-67 $\leq 14 \%$ ), luminal B/HER2-negative-like subtype (HR positive, HER2 negative, ki-67 > 14\%), luminal B/HER2-positive-like subtype (HR positive, HER2 positive), HER2-positive-like subtype (HR negative, HER2 positive) and triple negative subtype (HR negative, HER2 negative).

\section{Chemotherapeutic and MRI protocols}

The treatment plan was chosen by oncologists and explained to every patient. The initial evaluation of patients before NAC included a complete medical history, physical examination, complete blood work, chest X-ray, CT scan and bone scan. Clip titanium was placed in the tumour bed in all patients before starting chemotherapy, to identify the area of the primary tumour at the time of surgery. All patients with HER2-positive cancers received trastuzumab-based regimen as neoadjuvant therapy.

Tumour size was measured on the last MRI performed after completing NAC. The studies were done on a $1.5 \mathrm{~T}$ MRI scanner (Best, The Netherlands). The patient was placed in a prone position. Protocol in space-occupying lesions in the breast includes an axial T1-weighted sequence (TR: $494 \mathrm{msec}$, TE: $8 \mathrm{msec}$, number of acquired signals: 2, slice thickness: $3 \mathrm{~mm}$, interval: $0.3 \mathrm{~mm}$ ) and T2-weighted sequence (TR: $5000 \mathrm{msec}$, TE: 120 msec, number of acquired signals: 2, slice thickness: $3 \mathrm{~mm}$, interval: $0.3 \mathrm{~mm}$ ), followed by 3D T1-weighted fast spoiled gradient-echo dynamic sequence, selective excitation of water, (TR: 23 msec, TE: $5.7 \mathrm{msec}$, angle: $20^{\circ}$, slice thickness: 2 $\mathrm{mm}$ ) acquiring 6 series, one pre-contrast series and five consecutive post-contrast series at 90-second intervals. Contrast agent (Gd-DOTA, DOTAREM, Guerbet) was administered using a bolus injection ( $2 \mathrm{~mL} / \mathrm{s}$ ) at a dose of $0.1 \mathrm{mmol} / \mathrm{kg}$ followed by a bolus of saline solution $(20 \mathrm{~mL})$. All images are analysed at a workstation. Subtraction images between the without contrast stage and the $2^{\text {nd }}-3^{\text {rd }}-4^{\text {th }}$ $5^{\text {th }}$ post-contrast phase were obtained, which were interpreted with the help of specific programs for the analysis of contrast enhancement and time-signal intensity curves. 


\section{MRI assessment}

Assessment of response was based on changes in tumour size in the MRI contrast sequences. Tumour size was calculated by summing the maximum diameters of tumour enhancement on axial slices of MRI, as the Response Evaluation Criteria in Solid Tumours (RECIST). The absence of a clear enhancement indicates no residual cancer. The final response was defined as the change in size between the pre-treatment and post-treatment MRI. Response categories, based on radiological examination with contrast MRI, were classified as: (1) imaging complete response on MRI (iCR: no evidence of residual disease on posttreatment MRI); and (2) non-iCR: residual disease on posttreatment MRI.

\section{Histopathologic analysis}

Pathologic measurement of the tumour size was used as the "gold standard" and compared to the MRI-measured residual tumour size. Samples for histopathological examination were cut into $5 \mathrm{~mm}$ slices, fixed in $10 \%$ neutral-buffered formalin, trying to identify any lesion that corresponded with invasive carcinoma. If the tumour lesion was evident, it was included in its entirety for morphological study with hematoxylin and eosin (H\&E). If no evident tumour was found, the clip marker was identified, and slides from the block containing the marker as well as the adjacent blocks were examined. Tumour response after NAC was classified as (1) pCR: no residual invasive tumour in the breast on final pathology; and (2) non-pCR: presence of residual invasive cancer on final pathology. If any residual invasive disease, pathologic tumour size was determined by measuring the longest dimension of a sample stained with $\mathrm{H} \& \mathrm{E}$ and the number of blocks in which invasive tumour was detected.

\section{Statistical analysis}

A descriptive analysis of the variables included in the study was performed. Continuous variables were expressed as mean and standard deviation, and categorical variables were expressed as absolute values and percentages with their estimated $95 \%$ confidence interval. Comparison of means was performed using Student's t test or Mann-Whitney test and analysis of variance or Kruskal-Wallis test, as appropriate after checking normality with the Kolmogorov-Smirnov test. Association of qualitative variables was estimated using the Chi-square test. Pearson correlation analysis was used to com-
TABLE 1. Clinical and tumour characteristics

\begin{tabular}{|c|c|c|c|c|}
\hline CONTINUOUS VARIABLES & Mean & SD & Median & Range \\
\hline AGE (years) & 47.22 & 10.10 & 42.0 & $31.0-75.0$ \\
\hline BASAL TUMOR SIZE (cm) & 3.99 & 1.97 & 3.40 & $1.60-13.0$ \\
\hline CATEGORICAL VARIABLES & & $\mathrm{n}$ & $\%$ & $95 \% \mathrm{Cl}$ \\
\hline \multirow{4}{*}{ CLINICAL TUMOR STAGE } & $\mathrm{Tl}$ & 7 & 7.6 & $1.6-13.6$ \\
\hline & T2 & 69 & 75.0 & $65.6-84.4$ \\
\hline & T3 & 13 & 14.1 & $6.5-21.8$ \\
\hline & $\mathrm{T} 4$ & 3 & 3.3 & $0.7-9.2$ \\
\hline \multirow{2}{*}{ HISTOLOGICAL TYPE } & Ductal & 85 & 92.4 & $86.4-98.4$ \\
\hline & Lobular & 7 & 7.6 & $1.6-13.6$ \\
\hline \multirow{4}{*}{ HISTOLÓGICAL GRADE } & G1 & 9 & 10.0 & $3.2-16.8$ \\
\hline & G2 & 32 & 35.6 & $25.1-46.0$ \\
\hline & G3 & 49 & 54.4 & $43.6-65.3$ \\
\hline & NA & 2 & & \\
\hline \multirow{2}{*}{$\begin{array}{l}\text { HORMONAL RECEPTOR } \\
\text { STATUS }\end{array}$} & Positive & 60 & 65.2 & $54.9-75.5$ \\
\hline & Negative & 32 & 34.8 & $24.5-45.1$ \\
\hline \multirow{2}{*}{ HER2 STATUS } & Positive & 25 & 27.2 & $17.5-36.8$ \\
\hline & Negative & 67 & 72.8 & $63.2-82.5$ \\
\hline \multirow{5}{*}{ MOLECULAR SUBTYPE } & Luminal A & 11 & 12.0 & $4.8-19.1$ \\
\hline & Luminal B/HER2- & 35 & 38.0 & $27.6-48.5$ \\
\hline & Luminal B/HER2+ & 16 & 17.4 & $9.1-25.7$ \\
\hline & HER2+ & 9 & 9.8 & $3.2-16.4$ \\
\hline & Triple negative & 21 & 22.8 & $13.7-31.9$ \\
\hline \multirow{2}{*}{ PRE-NAC AXILLARY STATUS } & Positive & 76 & 82.6 & 74.3-90.9 \\
\hline & Negative & 16 & 17.4 & $9.1-25.7$ \\
\hline
\end{tabular}

$\mathrm{Cl}=$ confidence interval; $\mathrm{NA}=$ not available; $\mathrm{SD}=$ standard deviation

pare the MRI-measured and pathological tumour sizes. Linear regression model was used to analyse the diagnostic accuracy of MRI. $P \leq 0.05$ was considered significant. Basic statistical indicators to assess the accuracy of MRI in detecting residual disease after NAC were calculated. The efficacy of MRI was measured by the predictive values. True negative (TN) was defined as negative in both MRI and pathology; true positive (TP) was defined as positive in both MRI and pathology; false negative (FN) was defined as negative on MRI and positive on pathology; and false positive (FP) was defined as positive on MRI and negative on pathology. To assess the accuracy of MRI in detecting residual disease, sensitivity: TP / (TP + FN), specificity: TN / $(\mathrm{TN}+\mathrm{FP})$, positive predictive value (PPV): TP / $(\mathrm{TP}+\mathrm{FP})$, negative predictive value $(\mathrm{NPV}): \mathrm{TN} /$ $(\mathrm{TN}+\mathrm{FN})$, and overall accuracy: $(\mathrm{VN}+\mathrm{VP}) /(\mathrm{TP}+$ 


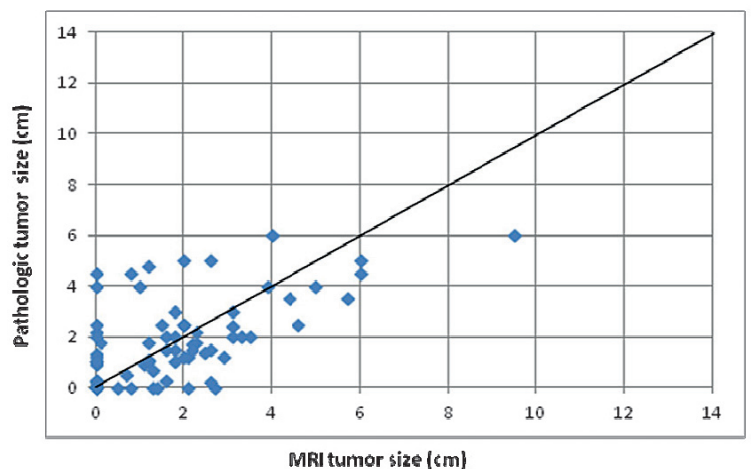

FIGURE 1. Dispersion graph of the correlation between residual tumour sizes $(\mathrm{cm})$ calculated at preoperative MRI (on the $X$-axis) and at pathological examination (on the Y-axis). Each point represents a tumour; if the pairs of data coincide, the points overlap. The bisector corresponds to the equivalence line. On the graph, the closer the points are to the equivalence line, the greater the correlation between MRI and pathology.

$\mathrm{TN}+\mathrm{FP}+\mathrm{FN}$ ) were calculated. Analyses were performed using IBM SPSS Statistics version 19.

\section{Results}

92 invasive breast tumours were analysed (1 patient with bilateral breast cancer was recorded). Initial clinicopathologic characteristics of patients and tumours of the study are shown in Table 1. Patients age ranged between 31 and 75 years (mean 47.22 years). Mean baseline tumour size determined by MRI was $3.99 \mathrm{~cm}$. Most tumours were diagnosed as T2 stage (75\%) and grade $3(53.3 \%)$. The initial biopsy of the lesions revealed 85 cases of invasive ductal carcinoma and 7 cases of invasive lobular carcinoma. 11 tumours were classified as luminal A, 35 as luminal B/HER2-, 16 as luminal B/HER2+, 9 as HER2+ and 21 as triple negative. Most patients (65.9\%) received taxane-anthracycline based chemotherapy; HER2-positive patients $(27.5 \%)$ were treated with a trastuzumab-based regimen. After NAC, mastectomy was performed in 21 cases $(21.8 \%)$ and BCS was attempted in 71 cases $(77.2 \%)$. Of the latter group, 16 cases were reoperated because tumour involvement or proximity to the resection margins.

\section{Accuracy of MRI to detect residual disease after NAC}

Tumour responses to NAC were compared based on the results obtained by MRI and pathological examination. MRI showed complete remission in
TABLE 2. MRI diagnostic performance in predicting pathologic response

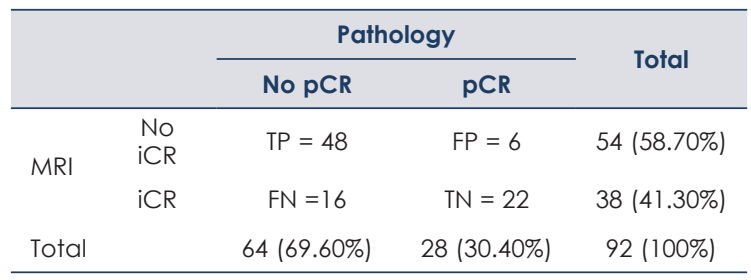

$\mathrm{FN}=$ false negative; $\mathrm{FP}=$ false positive; $\mathrm{iCR}=$ imaging complete response: $\mathrm{PCR}=$ pathologic complete response; $\mathrm{TN}=$ true negative; $\mathrm{TP}=$ true positive

38 cases $(41.3 \%)$ and residual disease in 54 cases (58.7\%). The pathological study showed a pCR in 28 cases $(30.4 \%)$ and invasive residual tumour was found in 64 samples (69.6\%).The diagnostic performance of MRI for detecting residual tumour is summarized in Table 2. The sensitivity of MRI for detecting residual disease after NAC was $75 \%$ $(48 / 64)$ and the specificity was $78.57 \%(22 / 28)$. The PPV (accuracy of MRI for detecting residual disease) was $88.89 \%$ (48/54). The NPV (accuracy of MRI in predicting pCR) was $57.89 \%(22 / 38)$. The overall accuracy of MRI was $76.09 \%$ (70/92). MRI showed FN diagnoses in $25 \%$ cases (16/64).

\section{Accuracy of MRI to predict the residual tumour size after NAC}

It was possible to compare the residual tumour size determined by MRI and pathological examination in 89 of the 92 cases of the study. In 3 cases it was not possible to determine the pathologic tumour size due to the presence of scattered residual multifocal disease. The mean residual tumour size determined by MRI after NAC was $1.44 \mathrm{~cm}$. The final pathologic tumour size was $1.53 \mathrm{~cm}$. The two measurements are correlated forwardly significantly $(\mathrm{r}=$ $0.648, \mathrm{p}<0.001$ ) (Figure 1). The mean discrepancy between the two measures was $0.96 \mathrm{~cm}$. The discrepancy was less than $1 \mathrm{~cm}$ in 57 cases (64.04\%).

\section{Analysis of factors influencing the accuracy of MRI for predicting residual tumour size.}

Linear regression models were performed to find clinicopathological predictors of the diagnostic accuracy of MRI based on the absolute difference between the MRI-measured and pathologic residual tumour size (Table 3). The strongest predictor was tumour grade $(p<0.001)$. The mean absolute 


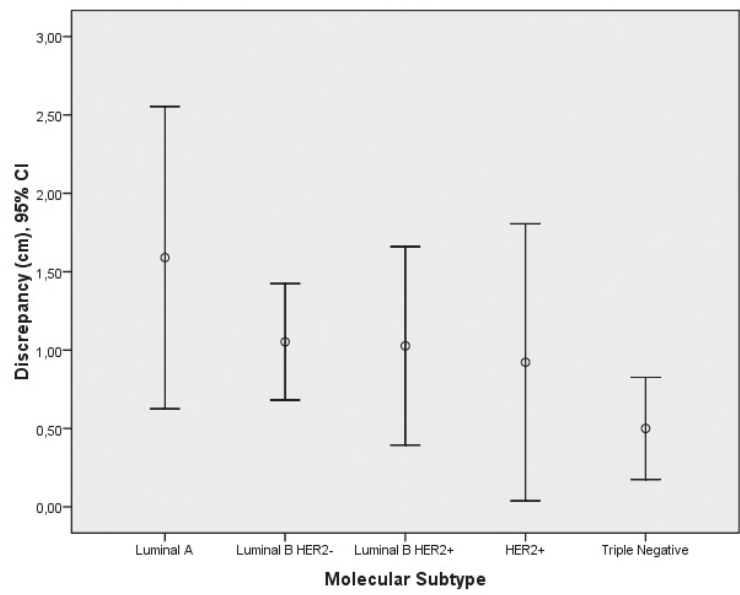

Discrepancy $(\mathrm{cm})$ based on molecular subtypes [Mean $\pm \mathrm{SD}$ ]:

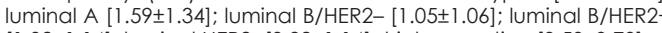
$[1.02 \pm 1.14]$; luminal HER $2+[0.92 \pm 1.14]$; triple negative [0.50 \pm 0.70$]$

FIGURE 2. Discrepancy between MRI-measured and pathologic residual tumour size, based on molecular subtypes.

discrepancy was significantly lower in the group of high-grade tumours. In addition, the HR status was associated significantly with the diagnostic accuracy of MRI, observing a lower discrepancy in the group of non-luminal tumours $(\mathrm{p}=0.033)$. Baseline tumour size was kept in the limit of significance, with a lower discrepancy in the group with baseline tumour size $\leq 5 \mathrm{~cm}$. The age, histological type and HER2 status were not associated with the diagnostic accuracy of MRI. Triple negative subtype showed the smallest difference between the two measurements (Figure 2), although no statistically significant difference regarding the molecular phenotype of the tumour $(p=0.055)$. After a multivariate linear regression analysis, tumour grade $(p=0.001)$ and baseline tumour size $(p=0.030)$ remained significant independent predictors of MRI accuracy (Table 4).

\section{Discussion}

Several researchers have previously studied the diagnostic accuracy of MRI in detecting invasive breast carcinoma in patients undergoing $\mathrm{NAC}^{31-}$ 34; however, an accurate determination of residual tumour size is necessary to perform an optimal surgery and achieve negative margins. We conducted a comparative analysis of post-NAC MRI and pathological findings to describe the diagnostic accuracy of MRI to detect residual invasive disease and to estimate the residual tumour size after NAC. In the current study, the overall diagnostic
TABLE 3. Factors affecting the MRI diagnostic accuracy based on the discrepancy between MRI and pathologic residual tumour size

\begin{tabular}{|c|c|c|c|}
\hline Variable & No. & $\begin{array}{l}\text { Discrepancy } \\
\text { (mean } \pm \text { SD) }\end{array}$ & $p$-value \\
\hline $\begin{array}{l}\text { Age (years) } \\
\quad \leq 45 \\
>45\end{array}$ & $\begin{array}{l}43 \\
46\end{array}$ & $\begin{array}{l}1.09 \pm 1.14 \\
0.84 \pm 1.01\end{array}$ & 0.281 \\
\hline $\begin{array}{l}\text { Baseline fumour size }(\mathrm{cm}) \\
\quad \leq 5 \\
>5\end{array}$ & $\begin{array}{l}74 \\
15\end{array}$ & $\begin{array}{l}0.85 \pm 0.99 \\
1.53 \pm 1.33\end{array}$ & 0.050 \\
\hline $\begin{array}{l}\text { Histological type } \\
\text { ductal } \\
\text { lobular }\end{array}$ & $\begin{array}{c}83 \\
6 \\
\end{array}$ & $\begin{array}{l}0.97 \pm 1.09 \\
0.87 \pm 0.82\end{array}$ & 0.818 \\
\hline $\begin{array}{l}\text { Histological grade } \\
1 \text { or } 2 \\
3\end{array}$ & $\begin{array}{l}40 \\
47\end{array}$ & $\begin{array}{l}1.44 \pm 1.24 \\
0.56 \pm 0.71\end{array}$ & $<0.001$ \\
\hline $\begin{array}{l}\text { Hormonal receptor status } \\
\text { positive } \\
\text { negative }\end{array}$ & $\begin{array}{l}59 \\
30 \\
\end{array}$ & $\begin{array}{l}1.14 \pm 1.13 \\
0.63 \pm 0.87\end{array}$ & 0.033 \\
\hline $\begin{array}{l}\text { HER2 status } \\
\text { positive } \\
\text { negative }\end{array}$ & $\begin{array}{l}24 \\
65\end{array}$ & $\begin{array}{l}0.99 \pm 1.12 \\
0.96 \pm 1.07\end{array}$ & 0.906 \\
\hline $\begin{array}{l}\text { Molecular subtype } \\
\text { Luminal A } \\
\text { Luminal B-HER2- } \\
\text { Luminal B-HER2+ } \\
\text { HER2+ } \\
\text { Triple negative }\end{array}$ & $\begin{array}{c}10 \\
34 \\
15 \\
9 \\
21\end{array}$ & $\begin{array}{l}1.59 \pm 1.34 \\
1.05 \pm 1.06 \\
1.02 \pm 1.14 \\
0.92 \pm 1.14 \\
0.50 \pm 0.70\end{array}$ & 0.055 \\
\hline
\end{tabular}

$S D=$ standard deviation

TABLE 4. Results from the Multivariate Regression Analysis

\begin{tabular}{lcccc}
\hline Variable & $\begin{array}{c}\text { Regression } \\
\text { coefficient (B) }\end{array}$ & se & $\mathbf{p}$ & $95 \% \mathrm{Cl}$ \\
\hline Tumour grade & 0.807 & 0.236 & 0.001 & $0.338-1.276$ \\
HR status & 0.086 & 0.249 & 0.729 & $-0.408-0.581$ \\
BTS (MRI) & 0.610 & 0.277 & 0.030 & $0.060-1.161$ \\
\hline
\end{tabular}

BTS = baseline tumour size $; \mathrm{Cl}=$ confidence interval $;$ se = standard error

accuracy of MRI for detecting residual invasive carcinoma in the breast was $76.09 \%$. The PPV and NPV were $88.89 \%$ and $57.89 \%$, respectively. These data suggest that breast MRI is an accurate tool for assessing tumour response after NAC, although it is more limited in predicting $\mathrm{pCR}$, which may be due to the NAC antiangiogenic effect in the tumour bed.

Correlation coefficients of residual tumour size assessed by MRI and pathology were considered good. Lobbes et al..$^{35}$ reviewed 17 studies comparing MRI-measured and pathologic residual tumour size. In this review, the mean correlation coefficient was 0.698 . In our series, the value of the correlation coefficient was 0.648 , reflecting a moderate correlation. If we remove the luminal A-like cases from 
the calculation, in which it has been observed that NAC is not the optimal therapeutic strategy, the value of global correlation coefficient rises to 0.706 $(p<0.001)$. Although correlation coefficients provide important information about the MRI's ability to assess response to NAC, the determination of the absolute difference between MRI-measured and pathologic residual tumour size is necessary to evaluate the diagnostic accuracy of MRI. MRI overestimation of residual tumour size can increase the number of unnecessary mastectomies and alter the cosmetic outcome of BCS with wide resection margins, while MRI underestimation can increase the number of reoperations. In the current study, the mean discrepancy was $0.96 \mathrm{~cm}$. Less than $1 \mathrm{~cm}$ difference in the calculation of residual tumour size between MRI and pathology was observed in 57 tumours (64.04\%). Identifying factors that may affect the accuracy of MRI for predicting residual tumour size could help to interpret breast MRI findings. Three recent studies evaluated the accuracy of MRI to predict residual tumour size after NAC and investigated the factors that influence the accuracy of MRI. In a study conducted by Ko et al. ${ }^{36}$, the Pearson's correlation coefficient between the tumour sizes measured using MRI and pathology was $0.749(p<0.001)$ and the mean of size discrepancy was $1.26 \mathrm{~cm}$. According to the molecular subtype, tumour grade and tumour morphology on initial MRI, statistically significant differences of size discrepancy between both measurements were observed. Triple negative subtypes were measured more accurately (mean, size discrepancy $=0.8 \mathrm{~cm}$ ). In the study by Chen et al..$^{37}$, the mean discrepancy was $1.0 \mathrm{~cm}$, and predictive factors found in the univariate analysis were histological type, tumour morphology, HR status, HER2 status and type of MRI. Multivariate analysis identified as independent predictors histological type, tumour morphology and the combination of HER2-HR status. Finally, in a study by Moon et al. ${ }^{38}$, the Pearson's correlation coefficient and the mean difference between MRI-measured and pathologic tumour size was $0.664(\mathrm{p}<0.001)$ and $1.39 \mathrm{~cm}$, respectively. The clinicopathological factors associated with MRI accuracy were the initial $\mathrm{T}$ stage, the age at the time of the diagnosis and the ER expression status. In addition, Moon et al observed increased accuracy of MRI in predicting the residual tumour extent after NAC in triple negative breast cancer. In our study, a statistically significant association was observed between the absolute discrepancy and each of covariates: baseline tumour size, tumour grade and HR status. A minor discrepancy was observed in tumours with an initial size $\leq 5 \mathrm{~cm}$, in high-grade tumours and in non-luminal tumours. Despite a statistically significant association between molecular subtypes and the diagnostic accuracy of MRI was not observed ( $p=0.055)$, a tendency to find better accuracy in triple negative tumours was found. The mean discrepancy in residual tumour size was lower in the group of triple negative tumours $(0.50 \mathrm{~cm})$. Multivariate analysis identified only as independent predictors baseline tumour size and tumour grade.

There are two important limitations to note in our study. The absence of ductal carcinoma in situ (DCIS) was not included in the definition of pCR, which can affect the accuracy of MRI. In addition, the current molecular classification includes a cutoff value of ki-67 expression level at 20\% to define low or high level. ${ }^{39}$

\section{Conclusions}

In conclusion, MRI can accurately measure tumour response and residual tumour size in breast cancer patients treated with NAC. Both overestimation and underestimation of MRI-measured residual tumour size may cause an incorrect surgical planning so it's important to consider the clinicopathological factors that can affect the diagnostic accuracy of breast MRI. In our series, evaluation of residual tumour size was more accurate in baseline tumour size $\leq 5 \mathrm{~cm}$ lesions, in high tumour grade lesions and in non-luminal breast cancer.

\section{References}

1. Hortobagy GN, Ames FC, Buzdar AU, Kau SW, McNeese MD, Paulus D, et al. Management of stage III primary breast cancer with primary chemotherapy, surgery, and radiation therapy. Cancer 1988; 62: 2507-16.

2. Mauriac L, Durand M, Avril A, Dilhuydy JM. Effects of primary chemotherapy in conservative treatment of breast cancer patients with operable tumors larger than $3 \mathrm{~cm}$ : Results of a randomized trial in a single centre. Ann Oncol 1991; 2: 347-54.

3. Schwartz GF, Birchansky CA, Komarnicky LT, Mansfield CM, Cantor RI, Biermann WA, et al. Induction chemotherapy followed by breast conservation for locally advanced carcinoma of the breast. Cancer 1994; 73: 362-9.

4. Calais G, Berger C, Descamps P, Chapet S, Reynaud-Bougnoux A, Body G, et al. Conservative treatment feasibility with induction chemotherapy surgery, and radiotherapy for patients with breast carcinoma larger than 3 $\mathrm{cm}$. Cancer 1994; 74: 1283-8.

5. Singletary SE, McNeese MD, Hortobagyi GN. Feasibility of breast-conservation surgery after induction chemotherapy for locally advanced breast carcinoma. Cancer 1992; 69: 2849-52.

6. Kuerer HM, Newman LA, Smith TL, Ames FC, Hunt KK, Dhingra K, et al. Clinical course of breast cancer patients with complete pathologic primary tumor and axillary lymph node response to doxorubicin-based neoadjuvant chemotherapy. J Clin Oncol 1999; 17: 460-9. 
7. van der Hage JA, van de Velde CJ, Julien JP, Tubiana-Hulin M, Vandervelden C, Duchateau L. Preoperative chemotherapy in primary operable breast cancer: results from the European Organization for Research and Treatment of Cancer Trial 10902. J Clin Oncol 2001; 19: 4224-37.

8. Bear HD, Anderson S, Brown A, Smith R, Mamounas EP, Fisher B, et al. The effect on tumor response of adding sequential preoperative docetaxel to preoperative doxorubicin and cyclophosphamide. Preliminary results from NSABP B-27. J Clin Oncol 2003; 21: 4165-74.

9. Bear HD, Anderson S, Smith RE, Geyer CE Jr, Mamounas EP, Fisher B, et al. Sequential preoperative or postoperative docetaxel added to preoperative doxorubicin plus cyclophosphamide for operable breast cancer: National Surgical Adjuvant Breast and Bowel Project Protocol B-27. J Clin Oncol 2006; 24: 2019-27.

10. Scholl SM, Fourquet A, Asselain B, Pierga JY, Vilcoq JR, Durand JC, et al. Neoadjuvant versus adjuvant chemotherapy in premenopausal patients with tumours considered too large for breast conserving surgery: Preliminary results of a randomized trial: S6. Eur J Cancer 1994; 30A: 645-52.

11. Fisher B, Bryant J, Wolmark N, Mamounas E, Brown A, Fisher ER, et al. Effect of preoperative chemotherapy on the outcome of women with operable breast cancer. J Clin Oncol 1998; 16: 2672-85.

12. Mauriac L, Macgrogan G, Avril A, Durand M, Floquet A, Debled M, et al. Neoadjuvant chemotherapy for operable breast breast carcinoma larger than $3 \mathrm{~cm}$ : a unicenter randomized trial with a 124 month median follow up. Am Oncol 1999; 10: 47-52.

13. Feldman LD, Hortobagyi GN, Buzdar AU, Ames FC, Blumenschain GR. Pathologic assessment of response to induction chemotherapy in breast cancer. Cancer Res 1986; 46: 2578-81

14. Chollet $P$, Charrier S, Brain E, Curé $H$, van Praagh I, Feillel V, et al. Clinical and pathological response to primary chemotherapy in operable breast cancer. Eur J Cancer 1997; 33: 862-6.

15. Kuerer HM, Newman LA, Buzdar AU, Dhingra K, Hunt KK, Buchholz TA, et al. Pathologic tumor response in the breast following neoadjuvant chemotherapy predicts axillary lymph node status. Cancer J Sci Am 1998; 4: 230-6.

16. Goldstein NS, Decker D, Severson D, Schell S, Vicini F, Margolis J, et al. Molecular classification system identifies invasive breast carcinoma patients who are most likely and those who are least likely to achieve a complete pathologic response after neoadjuvant chemotherapy. Cancer 2007; 110: 1687-96.

17. Abraham DC, Jones RC, Jones SE, Cheek JH, Peters GN, Knox SM, et al. Evaluation of neoadjuvant chemotherapeutic response of locally advanced breast cancer by magnetic resonance imaging. Cancer 1996: 78: 91-100.

18. Esserman L, Hylton N, Yassa L, Barclay J, Frankel S, Sickles E. Utility of magnetic resonance imaging in the management of breast cancer: evidence for improved preoperative staging. J Clin Oncol 1999; 17: 110-9.

19. Kuhl CK, Schmutzler RK, Leutner CC, Kempe A, Wardelmann E, Hocke A, et al. Breast MR imaging screening in 192 women proved or suspected to be carriers of a breast cancer susceptibility gene: Preliminary results. Radiology 2000; 215: 267-79.

20. Kriege $M$, Brekelmans $C T$, Boetes $C$, Besnard PE, Zonderland HM, Obdeijn IM, et al. Efficacy of MRI and mammography for breast-cancer screening in women with a familial or genetic predisposition. N Engl J Med 2004; 351: 427-37.

21. Berg WA, Gutiérrez L, NessAiver MS, Carter WB, Bhargaven M, Lewis RS, et al. Diagnostic accuracy of mammography, clinical examination, US, and MR imaging in preoperative assessment of breast cancer. Radiology 2004; 233: 830-49.

22. Schott AF, Roubidoux MA, Helvie MA, Hayes DF, Kleer CG, Newman LA et al. Clinical and radiologic assessments to predict breast cancer pathologic complete response to neoadjuvant chemotherapy. Breast Cancer Res Treat 2005; 92: 231-8.

23. Balu-Mastro C, Chapellier C, Bleuse A, Chanalet I, Chauvel C, Largillier R.. Imaging in evaluation of response to neoadjuvant breast cancer treatment benefits of MRI. Breast Cancer Res Treat 2002; 72: 145-52.

24. Bodini M, Berruti A, Bottini A, Allevi G, Fiorentino C, Brizzi MP, et al. Magnetic resonance imaging in comparison to clinical palpation in assessing the response of breast cancer to epirubicin primary chemotherapy. Breast Cancer Res Treat 2004; 85: 211-8.

25. Segara D, Krop IE, Garber JE, Wine E, Harris L, Bellon JR, et al. Does MRI predict pathologic tumor response in women with breast cancer undergoing preoperative chemotherapy? J Surg Oncol 2007; 96: 474-80.
26. Kim HJ, Im YH, Han BK, Choi N, Choi N, Lee J, Kim JH, et al. Accuracy of MRI for estimating residual tumor size after neoadjuvant chemotherapy in locally advanced breast cancer: relations to response patterns on MRI. Acta Oncol 2007; 46: 996-1003.

27. Fangberget A, Nilsen LB, Hole KH, Holmen MM, Engebraaten O, Naume B, et al. Neoadjuvant chemotherapy in breast cancer response evaluation and prediction to treatment using dynamic contrast-enhanced and diffusionweighted MR imaging. Eur Radiol 2011; 21: 1188-99.

28. Dongfeng H, Daqing D, Erhu J. Dynamic breast magnetic resonance imaging: pretreatment prediction of tumour response to neoadjuvant chemotherapy. Clin Breast Cancer 2012; 12: 94-101.

29. Nadrljanski MM, Milosevic ZC, Plesinac-Karapandzic V, Maksimovic R. MRI in the evaluation of breast cancer patient response to neoadjuvant chemotherapy: predictive factors for breast conservative surgery. Diagn Interv Radiol 2013; 19: 463-70.

30. Goldhirsch A, Wood WC, Coates AS, Gelber RD, Thürlimann B, Senn $\mathrm{HJ}$. Strategies for subtypes-dealing with the diversity of breast cancer: highlights of the St Gallen International Expert Consensus on the Primary Therapy of Early Breast Cancer 2011. Ann Oncol 2011; 22: 1736-47.

31. Straver ME, Loo CE, Rutgers EJT, Oldenburg HS, Wesseling J, Vrancken Peeters MJ, et al. MRI model to guide the surgical treatment in breast cancer patients after neoadjuvant chemotherapy. Ann Surg 2010; 251: 701-7.

32. Chen JH, Feig B, Agrawal G, Yu H, Carpenter PM, Mehta RS, et al. MRI evaluation of pathologically complete response and residual tumours in breast cancer after neoadjuvant chemotherapy. Cancer 2008; 112: 17-26.

33. Williams M, Eatrides J, Kim J, Talwar H; Esposito N, Szabuni M, et al. Comparison of breast magnetic resonance imaging clinical tumor size with pathological tumor size in patients status post-neoadjuvant chemotherapy. Am J Surg 2013; 206: 567-73.

34. McGuire KP, Toro-Burguete J, Dang H, Young J, Soran A, Zuley M, et al. MRI staging after neoadjuvant chemotherapy for breast cancer: does tumor biology affect accuracy? Ann Surg Oncol 2011; 18: 3149-54.

35. Lobbes MB, Prevos R, Smidt M, Tjan-Heijnen VC, van Goethem M, Schipper $R$, et al. The role of magnetic resonance imaging in assessing residual disease and pathologic complete response in breast cancer patients receiving neoadjuvant chemotherapy: a systematic review. Insights Imaging 2013; 4: $163-75$.

36. Ko ES, Han BK, Kim RB, Ko EY, Shin JH, Hahn SY, et al. Analysis of factors that influence the accuracy of magnetic resonance imaging for predicting response after neoadjuvant chemotherapy in locally advanced breast cancer. Ann Surg Oncol 2013; 20: 2562-8.

37. Chen JH, Bahri S, Mehta RS, Chen JH, Bahri S, et al. Impact of factors affecting the residual tuor size diagnosed by MRI following neoadjuvant chemotherapy in comparison to pathology. J Surg Oncol 2014; 109: 158-67.

38. Moon H-G, Han W, Ahn SK, Cho N, Moon WK, Im SA, et al. Breast cancer molecular phenotype and the use of HER2-targeted agents influence the accuracy of breast MRI after neoadjuvant chemotherapy. Ann Surg 2013; 257: $133-7$.

39. Goldhirsch A, Winer EP, Coates AS, Gelber RD, Piccart-Gebhart M, Thürlimann B, et al. Personalizing the treatment of women with early breast cancer: highlights of the St Gallen International Expert Consensus on the Primary Therapy of Early Breast Cancer 2013. Ann Oncol 2013; 24: 2206-23. 